\title{
CONSECUENCIAS PROFESIONALES DE LAS DEBILIDADES CONCEPTUALES EN EL ÁREA DE CONTROL
}

\author{
Walter Sánchez Chinchilla*
}

\footnotetext{
* Docente Facultad de Ciencias Económicas y Empresariales Universidad de Pamplona. Pamplona Colombia. E-mail: waltersanchex@hotmail.com
} 


\section{CONSECUENCIAS PROFESIONALES DE LAS DEBILIDADES CONCEPTUALES EN EL ÁREA DE CONTROL}

\section{RESUMEN}

Las debilidades conceptuales en control son, sin duda alguna, parte de las causales de las discusiones bizantinas entre la auditoría externa y la revisoría fiscal. Los problemas de formación inciden en la instrumentalización del ejercicio orientado a la estéril auditoría financiera, apoyado en los tristes celebres papeles de trabajo y con tan nulo impacto e incidencia social. Solo las normas amparan el ejercicio. No es lo mismo CONTROLAR, que EVALUAR y que hacer SEGUIMIENTO. Hasta ahora en la mayoría de los casos, la revisoría fiscal utiliza los rudimentos propios del concepto EVALUACIÓN y en algunos casos del de SEGUIMIENTO, pero no los de CONTROL. Es decir no se practica control pese a que unas normas particulares lo exigen y otras señalan una vereda diferente.

Palabras Claves: Control, Evaluación, Seguimiento.

\begin{abstract}
The conceptual weaknesses of control are, without a doubt, part of the causes of the byzantine discussions between the external auditor and the state reviewer. The problems of formation have their incidence in the instrumentation of the exercise oriented to the sterile financial audit supported by the sad, but famous work papers and with the null and void impact and social incidence. Only the norms aid in the exercise. It is not the same TO CONTROL as TO EVALUATE and then do a Follow-Up. Even now in the majority of the cases, the reviewer of the audit utilizes the rudimentary purposes of the EVALUATION concept and in some cases in the FOLLOW-UP but not those of CONTROL. This means that control is not practiced even though some particular norms demand this and others indicate a totally other difference.
\end{abstract}

Key Words: Control, Evaluation, Follow-U

Face $\operatorname{\text {IssN}} 1794-9920$

Recepción: Abril de 2008

Revisión: Mayo de 2008

Aceptación: Mayo de 2008 


\section{ENSEÑANZAS AFECTIVAS}

- ¿Alguna vez en su vida ha sufrido un accidente automovilístico siendo usted el conductor?

- ¿Ha sabido de algún accidente automovilístico de un familiar o conocido, yendo ellos al volante?

- ¿En alguna ocasión ha expresado, con respecto a algo ó a alguien: se me salió de control?

- ¿En alguna ocasión ha proferido el viejo refrán, "después del ojo afuera no hay Santa Lucía que valga?"

- ¿En algún momento de su vida se vio precisado a "montarle cacería" a algo o alguien?

- ¿Conoce a algún individuo que se le pueda calificar como de una vida caótica o desordenada, sin brújula?

- ¿Ha observado hechos sociales o ambientales anormales, inusitados?

- ¿Su presente profesional es producto del azar o, por el contrario, de la conjunción de decisiones y acciones?

El conductor de un vehículo transita por la gran ciudad de Medellín corroborando mentalmente que su tierra, definitivamente, es un jardín. Tantas mujeres bellas en las pasarelas de los andenes así lo indican. Después de sobrepasar un lindo rostro, se encuentra con un cuerpo espectacular, luego con una rubia encantadora y segundos antes de escuchar un estridente ruido y sentir un fuerte impacto, la flor más bonita ha cruzado la berma, una ex reina nacional de la belleza.

Con las manos asidas fuertemente al volante y con el rostro color arco iris, por unos segundos permanece impertérrito y confundido, sin saber que realmente sucedió. Con la misma competencia inconsciente sale del vehículo y con las manos en la cabeza - de reojo se fija que la ex reina ya no se encuentre - se dirige a la parte delantera de su vehículo donde es esperado por otro conductor iracundo quien vociferante le increpa por su irresponsabilidad. El vehículo colisionado es un deportivo Mercedes Benz modelo 2008, tan solo tiene una semana de uso. Las partes averiadas no admiten reparación, sino reemplazo; cada una de las modernas direccionales valen una cuantía superior a los seis dígitos. Mientras tanto, nuestro atontado, enamoradizo y desatento conductor no atina a balbucear palabra alguna pues, casualmente, el día anterior el seguro del vehículo había vencido.

Las lamentaciones y las autoincriminaciones se anidan en su mente. Uno de los transeúntes dice a otro: "eh ave maría, después del ojo afuera no vale santa lucía" y el otro le contesta: "Es que en esta avenida hay que manejar con cuatro ojos, mijo"

Agredido y agresor cuando los ánimos y la mente se asientan, se lamentan y EVALÚAN los daños, determinan la cuantía del siniestro y acuerdan las acciones para reencauzar la marcha normal. 
En otro lado de la vida cotidiana un padre de familia se halla al borde del abismo. Su hijo mayor se encuentra en el mundo de las drogas, la sospecha se volvió evidencia. Es inapelable. Madre y Padre se reclaman para si responsabilidades y méritos. El joven de un momento a otro "se le salió de las manos" y las posibilidades de volver a atrás son nulas, como las de recuperación son azarosas y sumamente costosas. En que momento y porqué nuestro hijo se nos perdió, se nos salió de las manos, es la interrogante que no deja dormir a los atribulados padres. ;Ahora no queda mas que internarlo y esperar!

En otro escenario del planeta, cientos de turistas que plácidamente disfrutan en una paradisiaca playa a la orilla del mar son sorprendidos por un monstruosa y gigantesca ola, el terreno pierde su estabilidad y sin capacidad de reacción son literalmente tragados por la naturaleza; después de unos eternos segundos, el mar recobra su normalidad y el desastre se evidencia. El hombre es impotente ante la madre naturaleza. Los por qué, abundan y nadie tiene una respuesta coherente. El ser superior es juzgado.

Finalmente, un empresario ha encontrado - tarde - que ha sido víctima de un robo continuo en el departamento de cartera. Sus premoniciones y desconfianza las había focalizado solamente en la Tesorería mediante mecanismos permanentes de seguimiento y la sorpresa ha sido mayúscula, la liebre ha saltado por un lugar insospechado.

Los casos anteriores nos muestran como la desatención, las externalidades, las limitaciones humanas, las concepciones erróneas, los imprevistos y la falta de brújula pueden dar al traste con sinnúmero de situaciones cotidianas, naturales $u$ organizacionales. Después de ello, el desorden, el caos y las lamentaciones afloran.

Trasladadas esas situaciones al ámbito profesional de los contadores públicos, nos ofrecen escenarios idénticos con repercusiones poco menos o tan desastrosas como las relatadas. El contador público ejerce una profesión de alto riesgo social. Su misión es de impacto social. Su rol en las sociedades ha sido, es y será - probablemente - medular. Lo probabilístico surge de su propio quehacer, le es imputable. Hasta ahora las normas lo amparan, pero ante el ambiente de desregulación que vive el planeta, ¿qué pasará?

Por si los problemas éticos fueran insuficientes y no mantuvieran atosigados de trabajo a la Junta Central de Contadores, por las investigaciones que cursan en contra de Contadores Públicos por ejercicio ilegal y violatorio del código de ética profesional, la presencia de un ejercicio ineficiente de las funciones de control externo están, años ha, a la orden del día y amenazando - como el volcán galeras - a la sociedad y al ejercicio profesional con lo cual le cercenaría uno de sus mas rentables frentes de ocupación.

Años ha, y como el volcán galeras, argumentos que en vez de arredrar mas bien incitan a no reflexionar en torno al quehacer profesional de la institución de la revisoría fiscal. 
Total la mayoría de quienes hoy ejercen la revisoría fiscal ostentan títulos posgraduales en ese campo y se supone han aprehendido los mas recientes avances en ella.

Un vuelo panorámico por las evidencias de ejercicio profesional sigue mostrando la presencia de los inveterados proforma de los papeles de trabajo propios de la auditoría financiera, que - a propósito, los estudiantes de posgrado aún exigen les sean "enseñados" (es que la memoria es traicionera, cierto); los dictámenes siguen conservando la añeja estructura argumental; el sistema de control interno se sigue evaluando - en el mejor de los casos - con cuestionarios de respuestas duales; Se evalúan los resultados, los informes de origen contable pero sin controlar el proceso que los arroja; se dictaminan, certifican y atestan, sobre todo, informes de carácter tributario; El enfoque del trabajo es meramente financiero y tributario, por aquello de las implicaciones sancionatorias; los informes se refieren solo al pasado, son de carácter normativo y casi siempre sus contenidos son sabidos por los administradores, quienes con desazón - como presintiendo que algo falta - los escuchan. En fin como era hace muchos años, aún sucede en los albores del siglo XXI; obviamente el adelanto es que los papeles de trabajo ya se diligencian en la computadora y no se usan los lápices de colores.

Los accionistas y empresarios cuentan con el revisor fiscal porque las normas lo exigen', pero preferirían tener a una figura que "agregara mas valor" y fuera menos apegado a lo meramente formal o contable; alcanzar una revisoría fiscal, en la mayoría de los casos, deviene mas del tráfico de influencias varias, que de la propuesta técnica; los mayores ejecutores, para "agregar valor" simultáneamente contratan otros servicios adicionales de asesoría y con ello "impactan"; los usuarios del servicio desconocen la filosofía de esta institución y también tienen debilidades conceptuales para exigir equis productos o para no coaccionar por otros; los contadores públicos en ejercicio de la revisoría fiscal se centran en los asuntos sencillos (el 10\% de la empresa) y descuidan los complejos e importantes $(90 \%)$.

Una acción de control de tercer nivel ${ }^{2}$ haría colapsar a la Junta Central de Contadores con los hallazgos provenientes de un ejercicio de la revisoría fiscal ajeno a su filosofía, contrario a las normas que tipifican las características del trabajo, sin evidencias documentales que demuestren unas adecuadas fases de planeación, ejecución, desarrollo y finalización del trabajo, con parcelación de las labores hacia lo meramente financiero, de carácter evaluativo más que de control, sin permanencia y oportunidad, ineficaz y parcializado, incapaz de contribuir a velar por la continuidad del ente económico, con

\footnotetext{
${ }^{1}$ Expresión permanente de presidentes y accionistas de grandes empresas.

2 Este tipo de control es el que ejerce, por ejemplo, la Auditoría General de la República sobre la gestión de las Contralorías en el nivel nacional y territorial.
} 
deprimentes evidencias físicas de las acciones y con los manidos y obsoletos papeles de trabajo de mero corte financiero ${ }^{3}$, entre otras.

Ojos que no ven, corazón que no siente Y MENTE QUE NO PIENSA ${ }^{4}$, agregaríamos para afirmar que las carencias conceptuales, con respecto al CONTROL, impiden la valoración o estimación de la práctica y, por ende, es un obstáculo para concebir instrumentos y metodologías de interventoría externa que satisfagan a todos los actores sociales que giran en torno a la revisoría fiscal.

No es lo mismo CONTROLAR, que EVALUAR y que hacer SEGUIMIENTO. Hasta ahora en la mayoría de los casos, la revisoría fiscal utiliza los rudimentos propios del concepto Evaluación y en algunos casos del de SEguimiento, pero no los de CONTROL. Es decir no se practica control pese a que unas normas ${ }^{5}$ así lo exigen y otras ${ }^{6}$ señalan una vereda diferente. Y, como no se practica control, de ahí se deriva el énfasis financierocontable y el desconocimiento de los asuntos estratégicos y de gestión de las organizaciones objeto de intervención externa. Así cualquiera puede hacer "revisoría fiscal"

En diferentes países desarrollados del planeta se han prendido las alarmas, y directas han quedado, ante la irrupción de grandes quiebras empresariales derivadas de las ambiciones desmedidas de los administradores, la correlativa corrupción y la laxitud por parte de individuos y de los sistemas de intervención externa ubicados, como era de esperarse, río abajo para ver los resultados, EVALUARLOS y "opinar" sobre ellos. Cientos de miles de pensionados, ahorradores, inversionistas, entre otros, vieron como sus recursos se desaparecieron como el agua entre las manos, de la noche a la mañana. Y los "vigilantes" no se percataron, no podían hacerlo, pues solo evaluaban, no controlaban. Es decir, cual empresa de seguridad los vigilantes solo se encargaron de manera estática de controlar el acceso al edificio mientras en su interior los delincuentes hacían de las suyas y cuando el vigilante se percató no encontró ni muebles.

La confianza desmoronada y el capitalismo amenazado de manera inusitada inducen a políticos y detentadores del poder a utilizar - ahora si conviene - el Estado para que este emita las normas tendientes a evitar que este tipo de situaciones se vuelva a presentar y formulan todo un conjunto de proposiciones derivadas todas del concepto CONTROL; Proposiciones que, de tiempos atrás, han sido consideradas obsoletas en nuestra legislación nacional y por algún sector profesional de la contaduría pública. Que vueltas da la vida, cierto.

\footnotetext{
${ }^{3}$ De allí la recurrente e ilegal práctica del dos por uno, contabilidad y revisoría fiscal en un solo paquete.

${ }^{4}$ Con el debido respeto a los limitados visuales, que pese a no poder ver, si miran con sus oídos y tacto.

${ }^{5}$ Entre ellos el código de comercio.

${ }^{6}$ La ley 43 de 1.990 con las "afamadas" normas de auditoría.
} 


\section{LAS DISCUSIONES DE BIZANCIO}

Bizantinas discusiones se han sostenido acerca de la auditoría y la revisoría fiscal las cuales se atizaron gracias a las debilidades conceptuales en esta temática. Son un hecho, verificable en el nivel de posgrados incluso, las debilidades en este campo. No saber diferenciar el control de otro tipo de prácticas de la misma taxonomía incide de manera directa en lo instrumental y desde luego que en la calidad del ejercicio profesional.

Se diseñan instrumentos apoyados en unos cimientos conceptuales débiles y de miope perspectiva pues, en el mejor de los casos, sus teorizaciones se circunscriben a las empresas y a lo meramente financiero. Esas concepciones encierran a los profesionales y no agregan valor a los usuarios.

$\mathrm{Al}$ desconocer el tinglado conceptual, las discusiones se dirimieron por la vía normativa a partir de correlación de poderes mas no de conocimientos. Era inapropiado comparar dos conceptos disimiles, cada uno con su vida propia, cada cual con su propia filosofía, y en ella cada una podía cumplir. Una, haciendo EVALUACIÓN y, la otra. practicando CONTROL. Esto último en teoría porque, ante la carencia de mentefactos conceptuales y la ausencia de producción intelectual ${ }^{7}$ propia del modelo de control de la tradición jurídica imperante en nuestro país, se recurrió a un frankeinstein: cuerpo de control y cabeza de evaluación. Las normas exigían desarrollar funciones de control pero las universidades dotaron a sus egresados de herramientas de evaluación meramente financiera ${ }^{8}$.

$\mathrm{Ni}$ rebajarse a pelear con un desnutrido Somalie ni osar a pelear con un fornido Vikingo. Agua y aceite. No había lugar a esa discusión mientras otros aprovecharon el río revuelto llevándose a la sociedad y a las empresas en esas aguas turbulentas, vituperando y perjudicando una de las más importantes instituciones y misiones del contador público. Esas son las circunstancias que permiten que cualquier recién egresado no dude en aceptar un cargo de "revisoría fiscal", en esas condiciones cualquiera lo puede hacer, solo evaluación financiera de carácter posterior, cero control para lo cual se requiere experiencia, conocimiento, bagaje y recorrido.

\footnotetext{
${ }^{7}$ Si bien es cierto el CP Rafael Franco Ruiz ha desarrollado, desde hace algunos lustros, su propuesta de control, esta ha carecido de su presentación escrita y difusión masiva para que fuese incorporado a los procesos de enseñanza-aprendizaje en las universidades; no obstante su reflexión pone en el mismo plano, como escuelas de control, a la Latina y a la Anglosajona, lo cual desde el punto de vista conceptual no es acertado, pues mientras la primera es CONTROL, la otra es EVALUACIÓN, conceptos totalmente diferentes. ${ }^{8}$ Una evidencia la podemos obtener en la corta antigüedad de la materia de Revisoría Fiscal y la larga ascendencia de las de Auditoría. Desde los primeros programas de contaduría de la década de 60 hasta la fecha, esas materias iniciaron y permanecen en los curriculum, e impertérritas y anonadadas las universidades, ni siquiera por ejercicio dialéctico, incorporan materias técnicas correspondientes con el Control. Los posgrados en Revisoría Fiscal tampoco se salvan, la mayoría solo tienen el título, pues imparten es Auditoría Externa.
} 
A oídos sordos palabras necias pues las discusiones no desembocaron en la producción de mentefactos y artefactos ${ }^{9}$ propios de la naturaleza del CONTROL produciendo en las organizaciones objeto de ello desazón y decepción ante el pálido aporte de la institución y de esta función esencial para la naturaleza y todo lo creado por el hombre.

\section{Enseñanzas cognitivas}

"Toda ciencia, metodología, o cualquier otro cuerpo de conocimientos se orienta hacia alguna estructura conceptual - un esquema de ideas reunidas para formar un total consistente o marco de referencia, al que se relaciona el contenido operacional de dicha área -. Sin tal estructura integrante, los procedimientos no pasan de ser rituales, sin sentido y carentes de razón o de sustancia; el progreso es solo una combinación afortunada de circunstancias; la investigación no es mas que una búsqueda a tientas en la oscuridad; y la diseminación del conocimiento es un proceso molesto, si en realidad hay algún "conocimiento" que transmitir" William J, Vatter

\section{MENTEFACTO DE CONTROL}

P1. El control es un subsistema del sistema de regulación ambiental.

P2a. El control se caracteriza porque es integral.

P2b. El control actúa de manera permanente.

P2c. El control tiene autonomía de acción.

P2d: El control es eficaz

P2e. El control es neutral.

P3a. Mientras que el control actúa de manera permanente, la evaluación lo hace a posteriori.

P3b. A diferencia del seguimiento que es focalizado el control es integral.

P4a. Dependiendo de su naturaleza los controles son de dos tipos: Naturales y Antrópicos.

P4b. Los controles naturales pueden ser bióticos o abióticos.

P4c. Dependiendo del objeto de control, los controles antrópicos se clasifican en sociales, económicos e ideológicos.

P4d. Dependiendo del ámbito de control, los controles sociales son: La Familia, El Estado y las Organizaciones ${ }^{10}$.

P4e. Dentro de los Controles Económicos y dependiendo del objeto de control tenemos: La Contabilidad, La Moneda y la Propiedad Privada.

\footnotetext{
${ }_{9}$ Salvo algunos desarrollos aplicados por la firma de contadores públicos Investigar Consultores S.A. en el Metro de Medellín y que mediante técnicas de control estratégico avizoró para esta empresa escenarios futuros a partir de los cuales sus directivos lograron oportunamente tomar decisiones correctivas.

${ }^{10} \mathrm{La}$ acepción hace referencia a organizaciones sociales con o son animo de lucro, contractuales o no contractuales.
} 
P4f. Los controles ideológicos, dependiendo de su objeto de control, son: La Cultura, La Religión y Los Modelos.

La clasificación que mayor difusión ha tenido en la profesión contable da origen a una taxonomía apropiadamente llamada por Mantilla como convencional y que resulta familiar para todo el mundo con el aditivo de que su utilidad práctica es muy reducida por tocar aspectos accesorios o accidentales del control (Mantilla). Como podrá sustraerse en la escala taxonómica propuesta, su perspectiva depende de los controles sociales en el nivel de Organizaciones y Estado, de allí la miopía que puede generar su fijación. las siguientes proposiciones las resumen:

P4g. Dependiendo de la relación con la organización objeto de control, este puede ser Interno o Externo.

P5h. Dependiendo de la función de la actividad organizacional sobre la cual actúa el control este se clasifica en: de legalidad, operacional, administrativo, de información, social.

P4i. Según la oportunidad se clasifica en: Previo, Perceptivo y Posterior.

Algunas de estas clasificaciones es importante analizarlas a la luz de las esencialidades del concepto con la posibilidad de que desaparezcan como tal, sin un proceso de filtración se plantean porque al fin y al cabo, son abordadas en la academia y en la cotidianidad. Su reflexión implicaría toda una estructura categorial no prevista en la presente.

Otro tipo de isoordinadas del concepto CONTROl y bajo el mismo enfoque planteado en este apartado, tiene que ver con la denominada por Mantilla como Técnico-Funcional y que resulta mas precisa aunque complicada y difícil su elaboración (Mantilla).

Dentro de esa taxonomía se pueden sustraer las siguientes proposiciones:

P4j. Según la naturaleza jurídica de las organizaciones el control puede ser: público o Privado.

P4k. En el sector público dependiendo del objeto de control se clasifica en: de Legalidad, Político, de Funciones, de Recursos y Administrativo.

Una clasificación del control proveniente de las ingenierías tiene que ver con la planteada por Ogata (1987) y su proposición es:

P4l. Según el sistema o tipo de control empleado el control se clasifica en: De lazo abierto, de lazo cerrado, adaptables y con aprendizaje.

Tal como lo reconoce Mantilla el control, sobre todo en el campo de la administración y la contaduria, ha estado bastante descuidado con las consecuencias por todos conocidas. 


\section{LA TEORÍA DEL CONTROL}

Desde el punto de vista rigurosamente conceptual, las anteriores son las proposiciones básicas del concepto CONTROL; esos son los cimientos que deben guiar la práctica profesional para entender, por ejemplo, como el Sistema de Control Interno se caracteriza por tener un alto contenido ideológico ${ }^{11}$ pues, al fin y al cabo, sus proposiciones se derivan del concepto matriz CONTROL. Entender esta proposición debe orientar a los contadores públicos, en funciones de control interno o externo, a diseñar técnicas e instrumentos que ausculten aspectos como la cultura, los afectos y las creencias, básicos dentro del ambiente de control de cualquier organización. Ello no se hace. Las interrogantes principales son documentales y procedimentales, e involucrando a los trabajadores, no a las personas. Se ve al empleado, no se mira a la persona. Esta situación deriva de una debilidad conceptual.

En sus importantes y pioneros planteamientos acerca del control, el Profesor Franco, a propósito de la cobertura del control, determina una serie de subsistemas (descomponiendo el todo en partes) dentro de una unidad económica como objetos de control - en coherencia con la proposición atrás relacionada con $\underline{\mathrm{P} 2 \mathrm{a}}$. - y planteando la oportunidad en tal ejercicio con respecto al pasado, al presente y al futuro - en concordancia con la proposición atrás relacionada con $\underline{\mathrm{P} 2 \mathrm{~b}}$. - no obstante, es una verdad de apuño que las prácticas profesionales en funciones de revisoría fiscal se dirigen fundamentalmente a lo financiero y al pasado. Es decir, tal pareciese, que los demás subsistemas no existiesen y que el tiempo no contara para las empresas y los sistemas de regulación ambiental como el CONTROL.

\begin{tabular}{|l|c|c|c|}
\hline \multicolumn{3}{|c|}{ Cobertura del Control12 } \\
\hline $\begin{array}{l}\text { OPORTUNIDAD/ } \\
\text { SUBSISTEMA }\end{array}$ & Futuro & Presente & Pasado \\
\hline Organización & $?$ & & $?$ \\
\hline Mercadeo & $?$ & & $?$ \\
\hline Inv. y Desarrollo & $?$ & & $?$ \\
\hline Producción & $?$ & & Auditoría Financiera \\
\hline Finanzas & $?$ & & $?$ \\
\hline Recursos Físicos & $?$ & & $?$ \\
\hline Recursos Humanos & $?$ & & \\
\hline
\end{tabular}

\footnotetext{
11 Sánchez C. Wálter. Control Interno Conceptual y Práctico. Investigar Editores.

12 Franco Ruiz Rafael. Seminario de Revisoría Fiscal. Material de Trabajo.
} 
¿Qué sentido tiene para una empresa, al final de un periodo, lamentarse de unos resultados negativos producto de unas pésimas decisiones, políticas o estrategias, o de estructuras organizacionales anacrónicas o de carencia de procesos de planeación, de investigación y desarrollo, o de mercadeo? ¿Qué sentido tiene para un empresario escuchar la formalidad de un dictamen que "descubre el agua tibia" de la quiebra de una empresa? Ningún. El control es permanente, integral, autónomo, eficaz y neutral y en esa perspectiva debe actuar quien cumple tales funciones.

Asumir la labor de sepulturero es una práctica propia de la Evaluación encarnada en la Auditoría Externa. La evaluación es posterior, se alimenta de informes, su preocupación es financiera - aunque ahora hablen de "auditoría integral" (expresión antitética) -. El Seguimiento es focalizado y, por consiguiente, poco eficaz, oneroso e inoportuno.

\section{Educación y ejercicio profesional}

La formación en el área de control ${ }^{13}$ es la fuente primigenia de las fallas en el ejercicio profesional. No se enseña - y por ende, no se aprende - CONTROL sino EVALUACIÓN. La materia de revisoría fiscal tiene, en la mayoría de los casos, un enfoque normativo ${ }^{14}$, con añejas metodologías, técnicas e instrumentos.

La teoría del CONTROL que se suministra en la mayoría de pregrados y posgrados es pobremente supraordinada ${ }^{15}$. Solo se concibe el CONTROL desde la perspectiva económica, un poco la social y con un enfoque instrumental ${ }^{16}$. Este bajo nivel de abstracción castra el pensamiento. Tan solo se acude a una CLASIFICACIÓN CONVENCIONAL ${ }^{17}$; Por ello se cree que solo existen dos tipos de control: privado y público; se confunden los conceptos de metodología, método, técnica, instrumentos, herramientas, entre otros.

Otros saberes como la biología, la misma economía, la sociología, expanden su acercamiento a sus objetos de estudio de una manera holística y abarcando mas allá de lo disciplinal, lo cual aumenta el espectro de conocimientos y por ende la posibilidad de re-crear mas conocimiento, nuevas teorías, modelos, métodos, metodologías, técnicas e instrumentos. Con esta mirada la miopía desaparece y los hallazgos pueden ser afortunados para la práctica profesional y para los usuarios del servicio, para la sociedad en general.

13 Con materias de Auditoría, algo así como Fidel Castro con un Buzo de Tommy.

14 ¿Será para saber cuales normas se violan o eluden?

15 Desde el punto de vista conceptual se refiere a la clasificación taxonómica del concepto, ver en el mentefactos de Control la proposición P1.

16 ¡El exacerbado interés en el hacer!

${ }^{17}$ Mantilla B. Samuel. Auditoría 2000. Ecoe ediciones. Página 74 


\section{Conceptualización y ejercicio del control}

Apoyados en las proposiciones básicas del concepto CONTROL, el ejercicio del mismo debe arraigar de una vez por todas que este implica permanencia, integralidad, autonomía, eficacia y neutralidad. La estructura categorial que implica desenmarañar cada uno de estos conceptos ha de servir para determinar los asuntos metodológicos e instrumentales.

Son solo tres los objetos de control dentro de una organización antrópica: el social, el económico y el ideológico, en otras palabras, la realidad y sus hechos y las personas y sus relaciones y las ideas; los mundos Popperianos. De suerte que, para cada uno de ellos, la Revisoría Fiscal debe diseñar metodologías, métodos, procedimientos, técnicas e instrumentos de control que PREVEAN, DETECTEN y CORRIJAN ${ }^{18}$.

A una organización la conforman las personas, sus recursos físicos y los pensamientos o ideas formalizadas o no; Cada uno de estos elementos es sustancial para la vida de las organizaciones, la evolución económica al girar las ha venido ubicando en lugares privilegiados; hoy las ideas o pensamientos, encapsulados en forma de conocimiento, son el insumo más valioso y diferenciador. ¿A través de cuáles métodos, procedimientos, técnicas e instrumentos de CONTROL el revisor fiscal se viene asegurando de su protección, de su conservación y de su continuidad? ¿A través de que medios el revisor fiscal se percata del impacto de los cambios ideológicos sociales y su incidencia en el mundo del trabajo y de las organizaciones? ¿A través de cuáles instrumentos el revisor fiscal devela oscuros intereses y cambios actitudinales en los individuos capaces de derrumbar a un ente económico? ¿Mediante que evidencias determina la calidad y cantidad de los controles administrativos y contables capaces de crear un ambiente favorable a los intereses económico-sociales de la empresa?

Si se trata de control, todas esas responsabilidades recaen sobre el agente que oficia en tales funciones, no para ejecutar las decisiones, sino para prevenir, detectar y determinar responsables a manera de corrección. La naturaleza tiene inmersa en si misma el control y como tal su sistema vela por el equilibrio de aquella, protegiéndose al máximo de los fenómenos, buscando su conservación y reaccionando cuando se vulnera de manera inusitada su funcionamiento gracias al depredador mayor: el hombre ${ }^{19}$. Como es allá en el mundo de los controles naturales ha de ser en el de los antrópicos y con más razón en ese microsistema como lo es una organización empresarial.

\footnotetext{
18 De ahí la característica de permanencia del control.

${ }^{19} \mathrm{La}$ naturaleza por el contrario, por su sabiduría, jamas ha "pensado" en la evaluación, la desconoce; prefiere evitar que lamentar. Seres bióticos y abióticos poseen solo sistemas de control. Los fenómenos que hoy sacuden al planeta son consecuencia de la acción depredadora del hombre y una defensa a su irracionalidad, no es parte de su sistema de regulación.
} 
La manipulación ideológica de la que viene siendo objeto la sociedad, da cuenta de la presencia de factores que tienden a alterar de diferentes maneras los roles de los individuos $\mathrm{y}$, mientras precisemos de su presencia en las organizaciones, deben ser objeto de estudio para diseñar sistemas de control que superpongan la naturaleza humana y respeten y defiendan sus derechos.

\section{Control y globalización}

El control en la investidura del revisor fiscal debe recordar su ascendencia y asumir la resistencia - como lo dice E. Sábato - para defender un modelo de intervención estatal pristino, plenamente vigente y en un momento histórico donde, unos cuantos, quieren a toda costa subyugar al Estado y a la sociedad en general en nombre del modernismo y la teoría del mercado. Si la revisoría fiscal recupera la memoria del modelo que le dio origen y de las bases conceptuales como institución de control, amaneceres radiantes se verán para la sociedad en general pues, su papel de regulación de los poderes ${ }^{20}$, es capaz de impedir que los intereses individuales se antepongan a los colectivos ${ }^{21}$.

El fenómeno globalizador ha servido para conocer, de primera mano, las consecuencias de un sistema de regulación como lo es la EVALUACIÓN, y que se ha concretado en las quiebras de grandes emporios económicos a lo largo de la geografía de los países capitalistas; Esos mismos escenarios han permitido conocer como en países como los EE.UU., se han percatado de estas anómalas situaciones y han estructurado normas ${ }^{22}$ orientadas a conjurarlas adoptando el modelo de CONTROL.

Los nuevos escenarios sociales, jurídicos y tecnológicos traen consigo enormes retos para el CONTROL y por ende para sus agentes. Reflexionar frente a los nuevos riesgos y amenazas organizacionales, económicas e informativas a la luz de los fundamentos teóricos ha de servir para proponer o re-crear novedosas prácticas de control que le permitan al conjunto de la sociedad conservar la imprescindible CONFIANZA PÚBLICA como valor social que lubrica el sistema vigente.

\section{A manera de conclusión}

Del pensamiento de Vatter expuesto en el aparte de enseñanzas cognitivas esta es nuestra conclusión: Sin tal estructura integrante (se refiere a los conceptos), los procedimientos no pasan de ser rituales, sin sentido y carentes de razón o de sustancia; el progreso es solo una combinación afortunada de circunstancias.

Hasta ahora la elaboración de papeles de trabajo en la practica indistinta de la Revisoría Fiscal o de la Auditoría Externa no pasa de ser eso rituales sin sentido y carentes de razón de allí que el progreso no se vea reflejado socialmente ni mas allá de los bolsillos de

\footnotetext{
${ }^{20}$ Franco Ruiz Rafael. Seminario de Revisoría Fiscal. Material de trabajo.

21 De ahí la característica de neutralidad del control.

22 Ver Ley Sarbanes-Oxley.
} 
quienes detentan el poder y el tráfico de influencias apoyados en la ignorancia propia y ajena, en la colectiva. Durante mas de cinco décadas la mayoría de nuestros colegas no hacen CONTROL tan solo practican EVALUACIÓN y en algunos casos (cuando hay siniestros) SEGUIMIENTO.

\section{Evaluación Afectiva}

1. ¿En calidad de empresario y accionista de una empresa y sabiendas que usted es contador público de profesión, qué le gustaría escuchar de labios del revisor fiscal?:

a. Que al cierre del ejercicio las utilidades fueron de $\mathrm{x}$ cantidad de unidades monetarias, según los saldos reflejados por los libros y resumidos en los estados financieros.

b. Que al cierre del ejercicio fiscal los saldos reflejados en los estados financieros han sido tomados fielmente de los libros oficiales registrados legalmente.

c. Que al cierre del ejercicio fiscal los resultados financieros son excelentes y que la empresa debe mejorar sus estrategias de largo plazo pues su continuidad se encuentra amenazada por factores exógenos.

d. Que al cierre del ejercicio fiscal los resultados financieros son excelentes y que la empresa pudo haber obtenido unos mejores dividendos de no mediar situaciones de elevación del gasto en investigación y desarrollo.

2. En calidad de padre de familia su hijo llama por celular para informarle que acaba de tener un accidente automovilístico. ¿Que le gustaría escuchar?:

a. Que el accidente se debió a impericia suya pero que no se preocupe porque solo hubo daños materiales.

b. Que el accidente se debió a impericia suya y el relato de los hechos y lamentaciones por lo que pudo haber sido y no fue.

c. Que el accidente se debió a impericia suya y que previamente tomó las previsiones del caso en cuanto a normas de tránsito, por lo cual la imputación de la culpa es a cargo del otro vehículo por violación de normas.

d. Que cree que el accidente se debió a impericia suya y que ya llamo a la aseguradora y arregló verbalmente con el otro conductor para no acudir a las autoridades de tránsito.

3. Ante una oportunidad o amenaza laboral usted generalmente actúa así:

a. Deja todo al azar y la suerte.

b. Deja todo en manos del ser superior.

c. Prospecta, prevé y diseña estrategias y acciones.

d. Espera que el hecho para lamentarse o regocijarse.

4. ¿En el ejercicio de una revisoría fiscal a la cual ha llegado con base en relaciones de amistad con uno de los accionistas que a su vez es el gerente de la empresa, sus acciones de fiscalización son?: 
a. Eventuales pues confía plenamente en el gerente y amigo.

b. Orientadas únicamente a lo contable pues el gerente se encarga del resto.

c. En función de los intereses del gerente pues este lo apoyó.

d. Ninguna de las anteriores.

\section{Evaluación Cognitiva}

Proposición 1. El control es un sistema de regulación ambiental junto con la Evaluación y el Seguimiento.

\section{Para cada proposición determine si se trata de Control (C), Evaluación (E), Seguimiento (S) o Ninguno (N).}

a. Los estados financieros reflejan la situación financiera de la empresa, de acuerdo a la revisión realizada con corte al 3112XX.

b. La evolución en el comportamiento de la cartera refleja un comportamiento preocupante por su crecimiento en volumen, cuantía y tipo de clientes.

c. __ El ambiente de control existente en la empresa es adecuado y contribuye al logro de los objetivos organizacionales.

d. __ De acuerdo a las evidencias obtenidas el faltante en inventarios se debe a un probable robo continuado con fecha indeterminada.

e. El arqueo de Caja no refleja evidencias que indiquen la existencia de irregularidades en el saldo.

f. Las evidencias obtenidas indican que en el año anterior se dejaron de cumplir con algunas obligaciones tributarias.

g. _ De acuerdo a lo observado, en los últimos seis meses el jefe de compras ha realizado compras a un reducido grupo de proveedores...

h. En nuestro criterio la administración debe abstenerse de autorizar transacciones crediticias que superen los montos autorizados por la Junta directiva.

\section{Proposición P4c. Dependiendo del objeto de control, los controles} antrópicos se clasifican en sociales, económicos e ideológicos.

2. Identifique cuáles de las siguientes proposiciones son verdaderas.

a. Los controles sociales son de origen ideológico y se orientan a controlar la riqueza.

b. La contabilidad es un sistema de control de origen económico.

c. La familia es un sistema de control ideológico que busca primigeniamente formar en los individuos roles y comportamientos adecuados a la sociedad. 
d. La aparición del concepto propiedad privada emerge como un sistema de control sobre los factores productores de renta.

e. El Estado emerge como una forma de control orientada a formar colectividades e identidades.

f. Los modelos concretan o idealizan representaciones que estandarizan hechos socioeconómicos de acuerdo a unos intereses.

g. La religión es un sistema de control social que influye en lo mas profundo de los individuos, sus creencias.

\section{REFERENCIAS}

- Franco R. Rafael. Seminario Revisoría Fiscal. Material y apuntes de trabajo.

- Sánchez c. Wálter. Control Interno conceptual y práctico. Investigar editores, segunda edición, 2006. 